\title{
Efectos de la cirugía bariátrica en resultados perinatales
}

\author{
Maritza Busquets $C .^{1}$, Magdalena Estefó A. ${ }^{a}$, Mónica Ferrero M. ${ }^{a}$, Soledad Silva B. ${ }^{a}$ \\ 1 Servicio Ginecología y Obstetricia, Hospital Parroquial de San Bernardo, Facultad de Medicina Universidad de los \\ Andes.
}

a Internas, Escuela de Medicina, Universidad de los Andes.

\section{RESUMEN}

Antecedentes: Según la OMS, la obesidad en el mundo se ha duplicado. En Chile, 50\% de las mujeres embarazadas tendrían sobrepeso u obesidad, lo que se asocia a condiciones de alto riesgo durante el embarazo. El creciente número de obesas en edad reproductiva y el bajo impacto de las medidas médicas para el control de peso, llevaron a considerar la cirugía bariátrica como opción terapéutica en el manejo de obesos refractarios a tratamiento convencional, mejorando las comorbilidades asociadas y calidad de vida. Diversos estudios retrospectivos han mostrado la eficacia de la cirugía bariátrica para disminuir complicaciones obstétricas asociadas a la obesidad, como diabetes gestacional e hipertensión. Sin embargo, existe información discordante con respecto a la cirugía bariátrica pre-concepcional y efectos adversos perinatales. Objetivo: Revisar y analizar la mejor evidencia disponible sobre la asociación entre cirugía bariátrica y resultados adversos perinatales. Método: Se realizó una búsqueda en los motores TripDatabase, Pubmed y Cochrane. Se establecieron criterios de inclusión y se seleccionaron artículos de acuerdo a las palabras clave "pregnancy", "pregnancy outcome", y "bariatric surgery". Resultados: Las pacientes embarazadas sometidas a cirugía bariátrica presentan menor riesgo de tener hijos GEG y mayor riesgo de PEG. No se observaron diferencias respecto a óbito fetal y malformaciones. Conclusión: La evidencia encontrada (tipo Ilb) orienta a que la cirugía bariátrica pre-concepcional se asocia a menor número de recién nacidos GEG y mayor número de PEG. Se requieren nuevos estudios que aporten un mayor nivel de evidencia y seguimientos a más largo plazo.

\section{PALABRAS CLAVES: Embarazo, resultados perinatales, obesidad, cirugía bariátrica}

\section{SUMMARY}

Background: According to the WHO, the prevalence of obesity has doubled worldwide. In Chile, $50 \%$ of pregnant women are overweight or obese. Obesity is associated to high risk pregnancies and higher Csection rates. The increasing number of obese women of reproductive age and the low impact of medical treatment for weight control have led to consider bariatric surgery as a therapeutic option after conventional treatment failure, improving quality of life and comorbidities. Several retrospective studies have shown the effectiveness of bariatric surgery in reducing obstetric complications associated to obesity, including gestational diabetes and hypertension. However, there is inconsistent evidence regarding pre-conceptional bariatric surgery and its relation to adverse neonatal outcomes. Objective: Review and analysis of the best available evidence relating to the association between bariatric surgery and adverse perinatal outcomes. Methods: TripDatabase, Pubmed and Cochrane search engines were used to find and select articles for analysis according to stablished inclusion criteria. The search was performed using the following key words: "pregnancy", "pregnancy outcome" and "bariatric surgery". Two studies where selected. Results: Current 
evidence shows that pregnant patients with previous bariatric surgery had lower risk of LGA and increased risk of SGA babies. No differences were found in relation to stillbirth and congenital abnormalities. Conclusion: The evidence analyzed shows that pre-pregnancy bariatric surgery is associated to lower rate of LGA and higher rate of SGA newborns. The evidence found was level Ilb. New studies are needed to validate this results, with better levels of evidence and long-term follow-up.

\section{KEY WORDS: Pregnancy, pregnancy outcomes, obesity, bariatric surgery}

\section{INTRODUCCIÓN}

De acuerdo con la Organización Mundial de la Salud (OMS), la obesidad se define como una enfermedad crónica, caracterizada por el aumento de la grasa corporal, asociada a un mayor riesgo para la salud. Desde 1980, las tasas de obesidad se han duplicado en todo el mundo (1).

De acuerdo con la Organización para la Cooperación y el Desarrollo Económicos (OCDE), Chile es el sexto país con más obesidad en el mundo (2). Según lo descrito en la Encuesta Nacional de Salud (ENS) 2010 , el $52,9 \%$ de las mujeres embarazadas que se controlan en el sistema de salud público tendrían algún grado de sobrepeso u obesidad. Las tasas varían según la edad, siendo de 7,5\% en embarazadas menores de 15 años, hasta un $31,1 \%$ en embarazadas entre los 35 y 44 años (3).

Además de los efectos negativos sobre la salud, la obesidad se asocia a diversas condiciones de alto riesgo durante el embarazo. La diabetes gestacional es entre 2,6 y 4 veces más frecuente y la pre-eclampsia aumenta entre 1,6 y 3,3 veces, duplicándose el riesgo por cada 5 a $7 \mathrm{~kg} / \mathrm{m} 2$ sobre el Índice de Masa Corporal (IMC) normal (4). Otros problemas asociados a la obesidad en el embarazo incluyen muerte fetal in útero, macrosomía fetal (recién nacido $>4000$ g), distocias del trabajo de parto y mayor tasa de cesáreas (5). En mujeres con sobrepeso y obesidad, la baja de peso preconcepcional debería reducir el riesgo de estas complicaciones (6).

La cirugía bariátrica se considera actualmente una opción terapéutica adecuada en el manejo de pacientes obesos refractarios a tratamiento convencional, asociándose significativamente a una disminución de comorbilidades y mejoría de la calidad de vida posterior a la intervención (7). Además, se ha determinado que se trata de una técnica costo-efectiva, logrando importantes beneficios para la salud a un precio relativamente aceptable (8).

Dentro de los distintos tipos de cirugía bariátrica, la más frecuente es el by-pass gástrico, seguido de la banda gástrica ajustable y la gastrectomía en manga $(9,10)$.

Se estima que cada año a nivel mundial se realizan más de 340.000 cirugías bariátricas (9). En nuestro país, no se conoce el número exacto de pacientes sometidos a esta intervención, sin embargo los estudios nacionales muestran un aumento progresivo de esta intervención $(11,12)$. El estudio de Guzmán y cols muestra un aumento progresivo de esta intervención desde 1990 hasta el año 2010 y que un $70 \%$ de las cirugías fueron realizadas en mujeres, con un promedio de edad de 37 años (12).

Dado el creciente uso de la cirugía bariátrica como tratamiento para la obesidad refractaria al manejo convencional y su uso en pacientes en edad fértil, es importante evaluar el impacto de ésta en el control obstétrico y resultados perinatales.

El objetivo de esta revisión es analizar la asociación de resultados adversos perinatales, especialmente en recién nacidos pequeños (PEG) y grandes para la edad gestacional (GEG), y cirugía bariátrica preconcepcional.

\section{MATERIALES Y MÉTODOS}

Se realizó una búsqueda durante septiembreoctubre de 2015 en las siguientes bases de datos online: Pubmed, Trip Database y Cochrane, utilizando los términos indexados "pregnancy", "pregnancy outcome", y "bariatric surgery".

Los criterios de inclusión fueron: ensayos clínicos, publicados entre Enero 2010 y Octubre 2015, escritos en español o inglés, con grupo control de mujeres embarazadas sin intervención, en que existiera registro de los resultados perinatales. Se excluyó trabajos que incluyeran embarazos múltiples o que no registrasen información sobre el peso pre-concepcional.

Se obtuvo 16 trabajos con las palabras clave definidas anteriormente, se seleccionaron 11 artículos según su título, los cuales se analizaron por resumen, dando así 4 trabajos que fueron analizados por completo según los criterios de exclusión e inclusión estipulados, para escoger finalmente dos que cumplían con las características descritas (Figura 1). Los últimos trabajos fueron descartados principalmente por fallas metodológicas. Se investigaron los siguientes outcomes primarios: Peso de nacimiento - PEG y GEG. Los resultados secundarios incluyen diabetes mellitus gestacional (DMG), preeclampsia, parto por cesárea, óbito y malformaciones congénitas. 

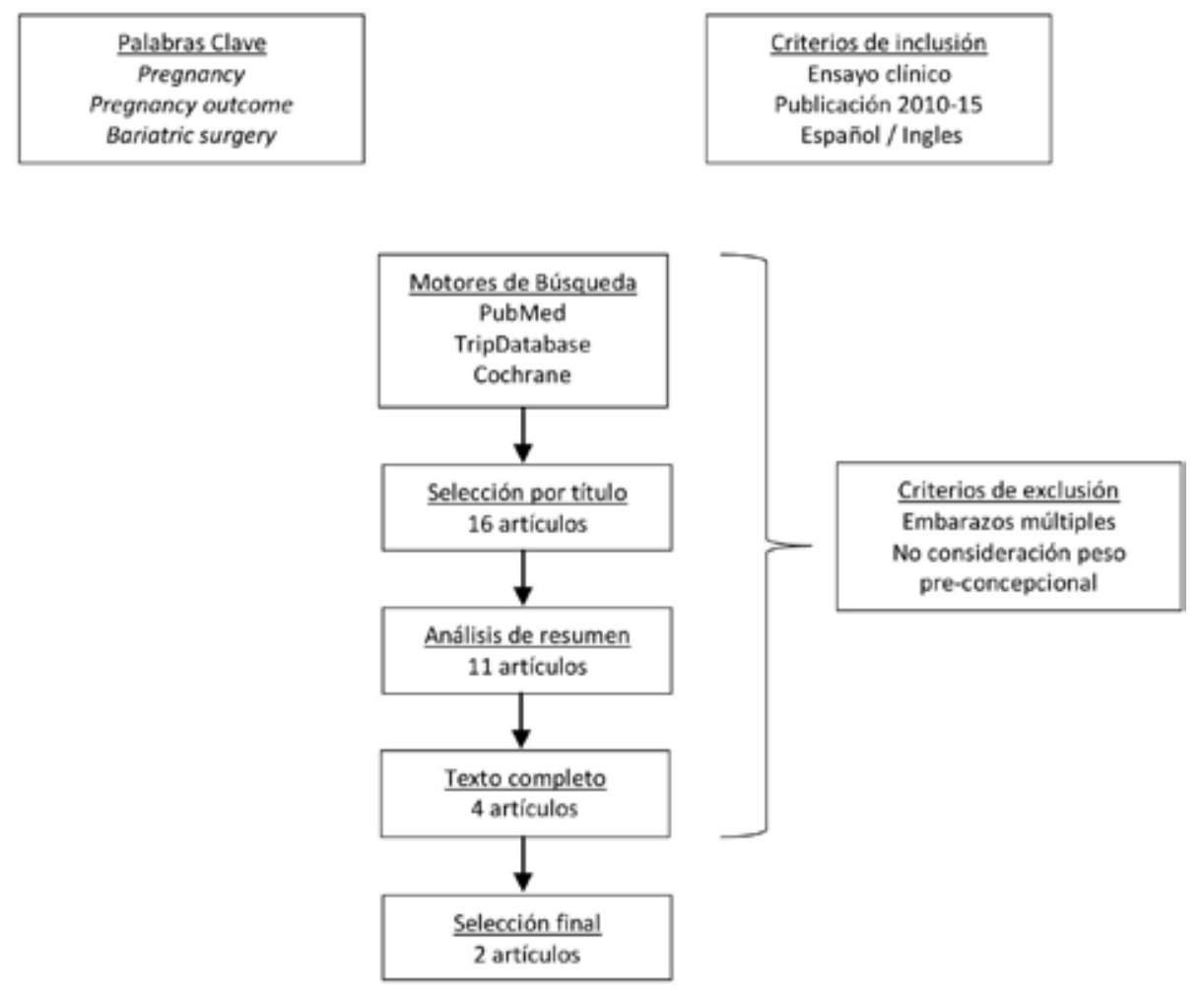

Figura 1. Metodología de búsqueda y selección de los manuscritos

\section{RESULTADOS}

Se seleccionaron dos estudios de cohorte retrospectivos que cumplían los criterios de inclusión $(13,14)$ (Tabla I).

Uno de los trabajos seleccionados (13), realizado en Suecia, obtuvo la información desde la base de dato nacional "Swedish Medical Birth Register", que abarca casi el $100 \%$ de la población y posee información del $98 \%$ de los nacimientos del país desde 1973 y de una segunda base de datos que contiene la información del $98,5 \%$ de todas las cirugías bariátricas realizadas en el país desde el 2007. Se analizaron los datos de 670 nacimientos de 616 mujeres embarazadas sometidas previamente a cirugía bariátrica $(98 \%$ de las intervenciones quirúrgicas fueron by-pass gástrico, $2 \%$ banda gástrica ajustable y menos de
$1 \%$ otros tipos de cirugía bariátrica disponible). Este grupo se comparó con un grupo control formado por emparejamiento de 1:5, para lo cual se tomó en cuenta la edad, paridad, IMC, (pre-quirúrgico en el grupo intervenido y precozmente durante el embarazo en el grupo control), tabaquismo, nivel educacional y año de parto. Los resultados primarios fueron: diabetes gestacional, definida como glicemia de ayuno $>126 \mathrm{mg} / \mathrm{dL}$ o PTGO $>180$ $\mathrm{mg} / \mathrm{dL}, \mathrm{GEG}$ : peso de nacimiento (PN) sobre el p90 para sexo y edad gestacional y PEG: PN bajo el p10 para sexo y edad gestacional. Otros resultados secundarios fueron bajo peso nacimiento (BPN; $<2,500 \mathrm{~g}$ ), macrosomía ( $>4,500 \mathrm{~g})$, nacimiento de pretérmino (<37 semanas), óbito fetal, muerte neonatal (antes de los primeros 28 días de vida), y malformación congénita mayor detectada el primer año de vida. 


\title{
Tabla I \\ RESUMEN DE RESULTADOS
}

\author{
Johansson et al. Outcomes of pregnancy after bariatric surgery (13) \\ - Menor riesgo de GEG en grupo intervenido (8,6\% vs. 22,4\%; OR 0,33; 95\%IC 0,24 a 0,44; p <0,001) \\ - Mayor riesgo de PEG en grupo intervenido (15,6\% vs. 7,6\%; OR 2,20; 95\% Cl 1,64 a 2,95; $p<0,001$ ). \\ - Menor diagnóstico DMG en grupo intervenido (1,9\% vs. 6,8\%; OR 0,25; 95\%IC 0,13 a 0,47; $p<0,001$ ). \\ - Sin diferencias significativas en relación a aumento de óbito fetal o malformaciones congénitas
}

\begin{abstract}
Kjær et al. The risk of adverse pregnancy outcome after bariatric surgery: a nationwide register-based matched cohort study (14)

- Menor riesgo de GEG en grupo expuesto (2,4\% vs. 7,3\%; OR 0,31; 95\%IC 0,15 a 0,65; $p<0,01)$

- Mayor riesgo de PEG (7,1\% vs. 2,9\%; OR 2,29; 95\%IC 1,33 a 3,96; $p<0,01)$.

- Sin diferencias significativas con respecto a diagnóstico de DMG, preeclampsia, inducción de trabajo de parto, cesárea, hemorragia postparto, Apgar 7 a los 5 minutos, admisión en UCl neonatal o muerte perinatal.
\end{abstract}

El segundo trabajo (14), fue realizado en Dinamarca, con datos obtenidos de dos bases de datos nacionales danesas ("The Danish National Patient Register" y "The Danish Medical Birth Registry"). La cohorte de intervención fue conformada por 339 mujeres con partos únicos entre 2004 y 2010 sometidas a cirugía bariátrica preconcepcional ( $83,4 \%$ by-pass gástrico y $16,6 \%$ banda gástrica). Para formar el grupo de control se realizó un emparejamiento 1:4 de embarazadas sin cirugía bariátrica (1.277 mujeres en total) de similares características en cuanto a IMC preconcepcional, edad materna, paridad y fecha de parto con respecto al grupo de estudio. Los resultados primarios fueron: partos de pretérmino, PEG (-2 DS del PN estimado según sexo y edad gestacional) y GEG (+2 DS del PN estimado según sexo y edad gestacional). Se consideraron como resultados secundarios: preeclampsia, DMG, inducción de trabajo de parto, cesárea, hemorragia postparto $(>500 \mathrm{ml})$, tiempo de hospitalización materna, Apgar $<7$ a los 5 minutos, admisión en $\mathrm{UCl}$ neonatal y muerte perinatal.

Recién nacidos GEG y PEG: En el estudio de Johansson y cols (13), el grupo intervenido se asoció a un menor riesgo de GEG (8,6\% vs. $22,4 \%$; OR 0,$33 ; 95 \%$ IC 0,24 a 0,$44 ; p<0,001)$ y macrosomía $(1,2 \%$ vs. $9,5 \%$; OR 0,$11 ; 95 \%$ IC, 0,05 a $0,24 ; p<0,001)$; y a mayor riesgo de PEG $(15,6 \%$ vs. $7,6 \%$; OR 2,20; $95 \% \mathrm{Cl} 1,64$ a 2,95; $p<0,001)$.

Los resultados fueron similares en el estudio de Kjaer y cols (14), donde se observó que los niños del grupo expuesto tuvieron menor peso de nacimiento (3.312 vs. $3.585 \mathrm{~g} ; \mathrm{p}<0,001)$, menor riesgo de GEG (2,4\% vs. 7,3\%; OR 0,31; $95 \%$ IC 0,15 a $0,65 ; p<0,01)$ y mayor riesgo de PEG $(7,1 \%$ vs. 2,9\%; OR 2,29; 95\%IC 1,33 a 3,96; $p<0,01$ ).
Otros resultados de interés: En el trabajo de Johansson y cols (13), se diagnosticó diabetes gestacional (a las 32 semanas de edad gestacional en promedio) en $1,9 \%$ de los embarazos posterior a cirugía bariátrica y $6,8 \%$ en los controles (OR 0,25; $95 \%$ IC 0,13 a 0,$47 ; p<0,001)$. En el estudio de Kjaer y cols (14) no hubo diferencias estadísticamente significativas entre los grupos (14).

En ambos estudios $(13,14)$, el promedio de tiempo transcurrido entre la cirugía bariátrica y el parto fue aproximadamente de 2 años. Johansson reporta que mientras mayor es el tiempo transcurrido entre la cirugía y el parto aumenta el riesgo de PEG y no encuentra mayores tasas de óbito fetal ni malformaciones congénitas en el grupo expuesto (13).

Kjaer y cols (14) no observan diferencias significativas con respecto a preeclampsia, inducción de trabajo de parto, cesárea, hemorragia postparto, Apgar $<7$ a los 5 minutos, admisión en UCI neonatal o muerte perinatal.

\section{DISCUSIÓN}

Múltiples estudios han mostrado la eficacia de la cirugía bariátrica para disminuir las complicaciones obstétricas asociadas a la obesidad, como la diabetes mellitus gestacional e hipertensión arterial (15). A pesar de los efectos beneficiosos que se han observado en el embarazo posterior a la cirugía bariátrica, surge la preocupación sobre las posibles consecuencias negativas secundarias a la restricción y/o malabsorción, que podrían llevar a nutrición y crecimiento fetal inadecuado. Varios estudios han mostrado menor peso de nacimiento en niños nacidos después de la cirugía bariátrica 
en relación con sus hermanos nacidos previo a la cirugía o a un grupo control (16).

Es conocido que la relación entre peso/talla del $\mathrm{RN}$ y la edad gestacional a la que nace tiene gran importancia clínica. El hecho de ser un RN PEG se asocia a un riesgo 5 veces mayor de muerte neonatal, dificultad en la termorregulación, hipoglicemia, hipocalcemia, poliglobulia e hiperbilirrubinemia. Dentro de las complicaciones asociadas al RN GEG además del trauma obstétrico, se encuentra una mayor mortalidad perinatal, hipoglicemia, poliglobulia, síndrome de distrés respiratorio (SDR) (síndrome aspirativo meconial / enfermedad de la membrana hialina), asfixia perinatal y efectos a largo plazo como síndrome metabólico e insulinorresistencia (independiente de la presencia de diabetes en la madre) (17).

En los dos trabajos presentados -ambos con una importante casuística- se pudo observar de forma consistente que las mujeres con antecedentes de cirugía bariátrica presentan mayor cantidad de RN PEG y menor cantidad de RN GEG que sus controles. A pesar que los resultados en ambos trabajos son estadísticamente significativos, los porcentajes de RN PEG observados en Kjaer y cols (14) no superan el $10 \%$, el cual es considerado una variación normal según su definición poblacional; por eso, se debe evaluar con cautela la significancia clínica de estos datos.

Los resultados obtenidos en estos artículos son concordantes con estudios anteriores al no encontrar diferencias significativas en cuanto a complicaciones perinatales, mortalidad perinatal, malformaciones congénitas y asfixia perinatal. $(18,19)$.

Respecto de la prevalencia de diabetes gestacional, sólo Johansson y cols (13) encuentran diferencias significativas entre ambos grupos. Nos parece importante mencionarlo por la alta prevalencia de esta patología en nuestra población, la que se aproxima a un $21 \%$ de acuerdo al estudio de Huidobro y cols (20).

Es necesario considerar que la obesidad es un factor de riesgo de enfermedades crónicas no transmisibles, las cuales pueden influenciar los resultados perinatales (21). Los resultados de ambos estudios presentados no fueron ajustados según la presencia o ausencia de hipertensión arterial, la que puede causar microangiopatía y por tanto aumentar independientemente el riesgo de restricción de crecimiento intrauterino (22).

Se ha descrito diferencia en el peso de los RN comparando grupos de cirugía malabsortiva y restrictiva. Kjaer y cols (14), realizan un análisis por subgrupos en el que se muestra que al incluir solo mujeres sometidas a by-pass gástrico ( $n$ : 286) comparándolas con sus respectivos controles, hay un mayor impacto en la diferencia de peso de nacimiento: $7,7 \%$ vs. $2,8 \%$ (AOR de 2,78 95\% IC 1,56 a 4,96 ) para PEG y $0,7 \%$ vs. $7,3 \%$ (AOR de 0,09 ;
95\%IC 0,02 a 0,36) para GEG (14). El estudio de Johannson y cols (13) no ajusta sus resultados según el tipo de cirugía realizada, pero vale la pena destacar que el $98 \%$ fue by-pass gástrico.

Ambos trabajos cumplieron con las características de un estudio metodológico de moderada-buena calidad, siendo evidencia tipo Ilb según Oxford (23). Sin embargo, las poblaciones evaluadas en estos trabajos (danesa y sueca) difieren de la población chilena, lo que influye negativamente en su validez externa.

Sería interesante, en investigaciones futuras, evaluar el efecto que podría tener la anemia secundaria a cirugía bariátrica malabsortiva en el embarazo y el neonato. Existen estudios como el de Devlieger y cols (24), que evalúa los niveles de micronutrientes posterior a cirugía batriátrica; entre los resultados obtenidos se observó que el peso de nacimiento era significativamente menor posterior a cirugías malabsortivas $(3,06 \pm 0,56 \mathrm{~kg})$ que restrictivas $(3,43 \pm 0,47 \mathrm{~kg})(p=0,024)$, y que tanto el grupo de cirugía restrictiva como malabsortiva mostró niveles disminuidos de micronutrientes maternos, efecto que tiende a ser más marcado en el grupo de cirugías malabsortivas. Sin embargo, dicho estudio carece de grupo control y presenta una cohorte de tamaño limitado (49 mujeres), por lo que se requiere mayor investigación al respecto.

\section{CONCLUSIÓN}

La evidencia encontrada (tipo Ilb), orienta a que la cirugía bariátrica pre-concepcional se asocia a menor número de recién nacidos GEG y mayor número de PEG. Se requieren nuevos estudios que aporten un mayor nivel de evidencia y seguimientos a más largo plazo.

\section{REFERENCIA}

1. WHO. Obesity: preventing and managing the global epidemic. Report of a WHO Consultation. WHO Technical Report Series 894. Geneva: World Health Organization [Internet] 2000. Disponible en: http:// www.who.int/nutrition/publications/obesity/WHO_ TRS_894/en/.

2. Organización para la Cooperación y el Desarrollo Económicos. Estadísticas de la OCDE sobre la salud 2014- Chile en comparación [Internet]. 2014. Disponible en: http://www.oecd.org/els/health-systems/ Briefing-Note-CHILE-2014-in-Spanish.pdf.

3. Ministerio de Salud. Encuesta Nacional de Salud ENS 2009-2010.[Internet]. Disponible: http://www.redsalud.gov.cl/portal/docs/page/minsalcl/g_home/ submenu_portada_2011/ens2010.pdf.

4. Weiss JL, Malone FD, Emig D, Ball RH, Nyberg DA, Comstock $\mathrm{CH}$, et al. Obesity obstetrics complications and cesarean delivery rate -a population- based screening study. ACOG 2004;190(4):1091-7.

5. Ross N, Neovius M, Cnattingius S, Trolle $Y$, Sääf $M$, Granath $\mathrm{F}$, et al. Perinatal outcomes after bariatric 
surgery: nationwide population based matched cohort study. BMJ 2013;347:f6460.

6. Ovesen P, Rasmussen S, Kesmodel U. Effect of prepregnancy maternal overweight and obesity on pregnancy outcome. Obstet Gynecol 2011;118:305-12.

7. Atalah E, Castro R. Obesidad materna y riesgo reproductivo. Rev Med Chile 2004;132:923-30.

8. Livingston $\mathrm{EH}$. Lowering the bariatric surgery minimum body mass index threshold. Arch Surg 2008;143(7):708-10.

9. Buchwald $\mathrm{H}$, Oien DM. Metabolic/bariatric surgery Worldwide 2008. Obes Surg 2009;19(12):1605-11.

10. Eldar S., Heneghan HM., Brethauer SA., Schauer PR. Bariatric surgery for treatment of obesity. Int $\mathrm{J}$ Obes (Lond) 2011;35 (3):S16-21.

11. Csendes A, Maluenda F. Morbimortalidad de la cirugía bariátrica. Experiencia chilena en 10 instituciones de salud. Rev Chil Cir 2006;58(3);208-12.

12. Guzmán S, Manrique M, Raddatz A, Norero E, Salinas $\mathrm{J}$, Achurra $\mathrm{P}$, et al. Results of bariatric surgery. Experience over 18 years. Rev Med Chil 2013;141(5):55361.

13. Johansson K, Cnattingius S, Näslund I, Roos N, Trolle Lagerros $\mathrm{Y}$, Granath $\mathrm{F}$, et al. Outcomes of pregnancy after bariatric surgery. N Engl J Med 2015;372(9):81424.

14. Kjær MM, Lauenborg J, Breum BM, Nilas L. The risk of adverse pregnancy outcome after bariatric surgery: a nationwide register-based matched cohort study. Am J Obstet Gynecol 2013;208:464.e1-5.

15. Sheiner E, Wilis K, Yogev Y. Bariatric surgery: impact on pregnancy outcomes. Curr Diab Rep 2013;13(1):19-26.
16. Aricha-Tamir B, Weintraub AY, Levi I, Sheiner E. Downsizing pregnancy complications: a study of paired pregnancy outcomes before and after bariatric surgery. Surg Obes Relat Dis 2012;8:434-9

17. Gormaz M. Pequeño para la Edad Gestacional en el periodo neonatal. Rev Esp Endocrinol Pediatr 2012;3(2):90-2.

18. Sheiner E, Levy A, Silverberg D, Menes T, Levy I, Katz M, et al. Pregnancy after bariatric surgery is not associated with adverse perinatal outcome. Am J Obstet Gynecol 2004;190:1335-40.

19. Maggard MA, Yermilov I, Li Z, Maglione M, Newberry $S$, Suttorp $M$, et al. Pregnancy and fertility following bariatric surgery: a systematic review. JAMA 2008;300(19):2286-96.

20. Huidobro A, Fulford A, Carrasco E. Incidencia de diabetes gestacional y su relación con obesidad en embarazadas chilenas. Rev Méd Chile 2004;132:931-8.

21. Farías M. Obesidad materna: severo problema de salud pública en Chile. Rev Chil. Obstet Ginecol 2013;78(6):409-12.

22. Bertoglia P, Rivas A, Navarrete P, Castro L, Acurio J, Escudero C. Resultados clínicos y perinatales de los embarazos con hipertensión arterial en un hospital de referencia de la VIII región de Chile. Rev Chil Obstet Ginecol 2010;75(3):162-71.

23. Manterota C, Zavando D. Cómo interpretar los "Niveles de Evidencia" en los diferentes escenarios clínicos. Rev Chil Cir 2009;61(6):582-95.

24. Devlieger R, Guelinckx I, Jans G, Voets W, Vanholsbeke $C$, Vansant $G$. Micronutrient levels and supplement intake in pregnancy after bariatric surgery: a prospective cohort study. PLoS ONE 2014;9(12):e114192. 